\title{
Open-loop deep brain stimulation for the treatment of epilepsy: a systematic review of clinical outcomes over the past decade (2008-present)
}

\author{
James J. Zhou, MD, Tsinsue Chen, MD, S. Harrison Farber, MD, Andrew G. Shetter, MD, and \\ Francisco A. Ponce, MD
}

Department of Neurosurgery, Barrow Neurological Institute, St. Joseph's Hospital and Medical Center, Phoenix, Arizona

OBJECTIVE The field of deep brain stimulation (DBS) for epilepsy has grown tremendously since its inception in the 1970s and 1980s. The goal of this review is to identify and evaluate all studies published on the topic of open-loop DBS for epilepsy over the past decade (2008 to present).

METHODS A PubMed search was conducted to identify all articles reporting clinical outcomes of open-loop DBS for the treatment of epilepsy published since January 1, 2008. The following composite search terms were used: ("epilepsy" [MeSH] OR "seizures" [MeSH] OR "kindling, neurologic" [MeSH] OR epilep* OR seizure* OR convuls*) AND ("deep brain stimulation" [MeSH] OR "deep brain stimulation" OR "DBS") OR ("electric stimulation therapy" [MeSH] OR "electric stimulation therapy" OR "implantable neurostimulators" [MeSH]).

RESULTS The authors identified 41 studies that met the criteria for inclusion. The anterior nucleus of the thalamus, centromedian nucleus of the thalamus, and hippocampus were the most frequently evaluated targets. Among the 41 articles, 19 reported on stimulation of the anterior nucleus of the thalamus, 6 evaluated stimulation of the centromedian nucleus of the thalamus, and 9 evaluated stimulation of the hippocampus. The remaining 7 articles reported on the evaluation of alternative DBS targets, including the posterior hypothalamus, subthalamic nucleus, ventral intermediate nucleus of the thalamus, nucleus accumbens, caudal zone incerta, mammillothalamic tract, and fornix. The authors evaluated each study for overall epilepsy response rates as well as adverse events and other significant, nonepilepsy outcomes.

CONCLUSIONS Level I evidence supports the safety and efficacy of stimulating the anterior nucleus of the thalamus and the hippocampus for the treatment of medically refractory epilepsy. Level III and IV evidence supports stimulation of other targets for epilepsy. Ongoing research into the efficacy, adverse effects, and mechanisms of open-loop DBS continues to expand the knowledge supporting the use of these treatment modalities in patients with refractory epilepsy. https://thejns.org/doi/abs/10.3171/2018.5.FOCUS18161

KEYWORDS DBS; deep brain stimulation; epilepsy; seizures; Stimulation of the Anterior Nucleus of the Thalamus for Epilepsy (SANTE)

$\mathrm{E}$ PILEPSY affects around 50 million people worldwide; 58 it is estimated that $30 \%$ to $40 \%$ of these patients are medically refractory to treatment. ${ }^{26,27,41}$ It is well documented that uncontrolled seizures cause significant morbidity and mortality, ${ }^{45}$ and while a large portion of these patients are candidates for surgical resection, a critical need exists for alternative treatment modalities for those who are not. In recent years, neurostimulation for the treatment of medically refractory epilepsy has gained considerable interest among patients and providers..$^{14,22,28,46,47}$

Deep brain stimulation (DBS) for the treatment of medically refractory epilepsy was pioneered in the 1970s and 1980 s, with early studies on the effects of cerebellar and anterior thalamic stimulation in patients with epilepsy. ${ }^{8,9,50}$ Since then, a growing body of literature has further sup-

ABBREVIATIONS ANT = anterior nucleus of the thalamus; $C M T=$ centromedian nucleus of the thalamus; $\mathrm{DBS}=$ deep brain stimulation; $\mathrm{HCP}=$ hippocampus .

SUBMITTED March 30, 2018. ACCEPTED May 11, 2018.

INCLUDE WHEN CITING DOI: 10.3171/2018.5.FOCUS18161. 


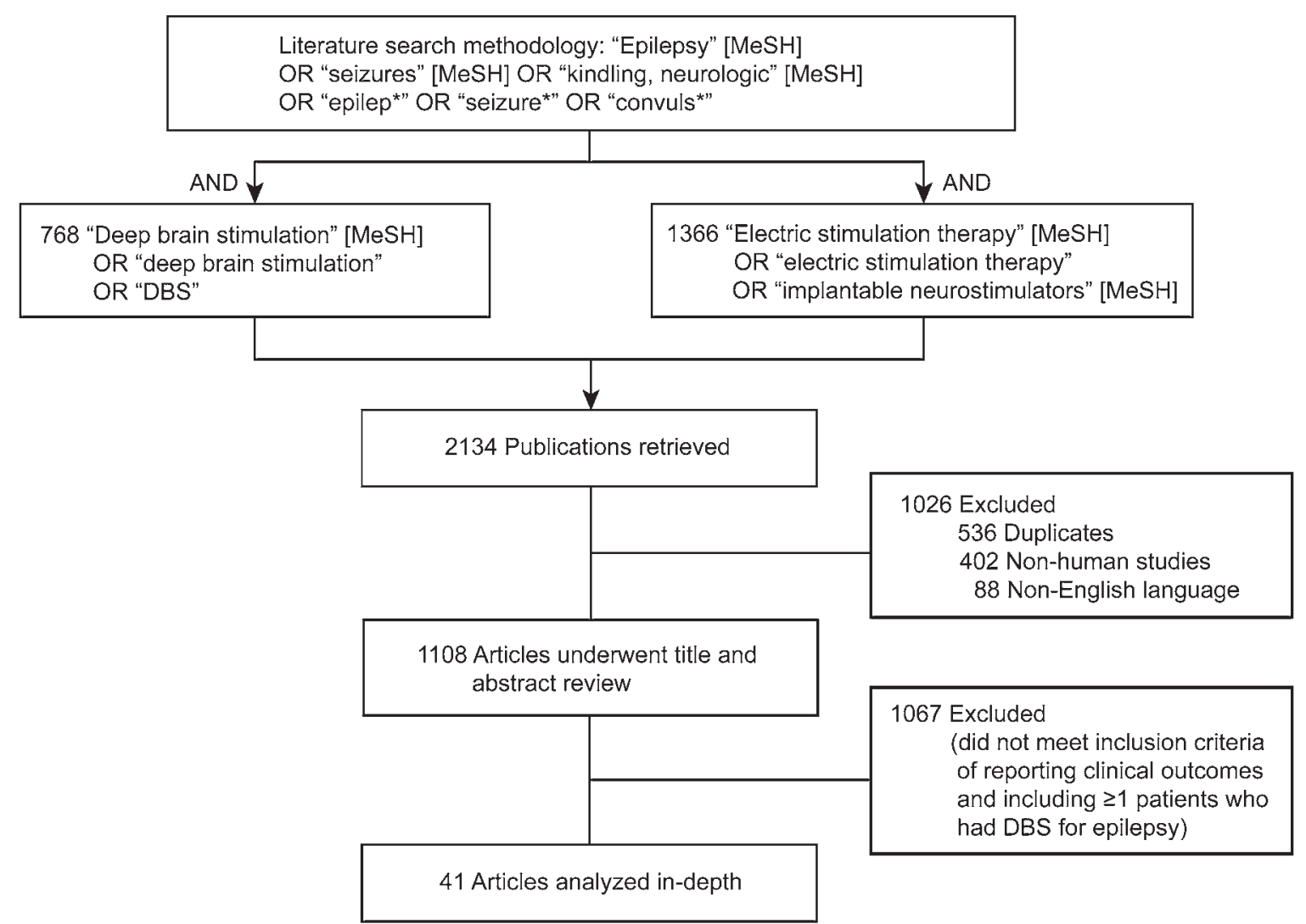

FIG. 1. Flowchart depicting literature search keywords, methodology, and number of studies found for clinical outcomes of openloop DBS for the treatment of epilepsy.

ported the safety and efficacy of established targets such as the anterior nucleus of the thalamus (ANT), while simultaneously exploring alternative targets such as the centromedian nucleus of the thalamus (CMT), and the hippocampus (HCP) ${ }^{6,24,25,35,52}$ This review focuses on studies of clinical outcomes of open-loop DBS published during the last 10 years.

\section{Methods}

\section{Search Methodology}

MEDLINE and PubMed inquiries of all studies reporting clinical outcomes of open-loop DBS since January 1, 2008, were performed (Fig. 1). Articles were included if they reported on at least one patient who underwent DBS for epilepsy and reported clinical outcomes. Articles were excluded if they reported nonclinical outcomes (i.e., electrographic or imaging findings), included nonhuman subjects, or were not published in English.

The following composite search terms were used: ("epilepsy" [MeSH] OR "seizures" [MeSH] OR "kindling, neurologic" [MeSH] OR epilep* OR seizure* OR convuls*) AND ("deep brain stimulation" [MeSH] OR "deep brain stimulation" OR "DBS") OR ("electric stimulation therapy" [MeSH] OR "electric stimulation therapy" OR "implantable neurostimulators" [MeSH]). The composite search term yielded 2134 articles. Of these, 536 articles were excluded because they were duplicates and 402 ar- ticles were excluded because they did not deal with human subjects. Another 88 non-English language publications were excluded, yielding a total of 1108 articles for final review. A title and abstract review, followed by a full-text review of selected articles, was subsequently conducted to identify publications for final inclusion. We evaluated each study for overall epilepsy response rates as well as for adverse events and other significant nonepilepsy outcomes.

\section{Results}

\section{Overall Findings}

Of the 1108 articles identified in the literature search, 41 articles met the inclusion criteria of both 1) reporting clinical outcomes and 2) including at least one patient who underwent DBS for epilepsy. The remaining 1067 articles were excluded on the basis of not meeting one or both of these criteria. Results of all 41 studies are summarized in Table $1^{1-6,10-13,15-17,19,20,23-25,29-40,42,44,48,49,51-57}$ and the most frequently studied stimulation targets were the ANT (20 studies $1,5,11,15,16,19,25,29,30,32,34,37-40,48,49,53-55)$, the CMT (7 studies $\left.^{10,11,31,44,51-53}\right)$, and the HCP (10 studies $^{3,4,11,12,13,20,33,35,36,56}$ ). It is noteworthy that some articles evaluated multiple targets and are included more than once. The remaining 7 articles $^{2,6,17,23,24,42,57}$ investigated alternative targets. The most relevant studies for ANT, CMT, and HCP are discussed in further detail in the following sections. As commonly defined in the literature, a clinical responder refers to a pa- 


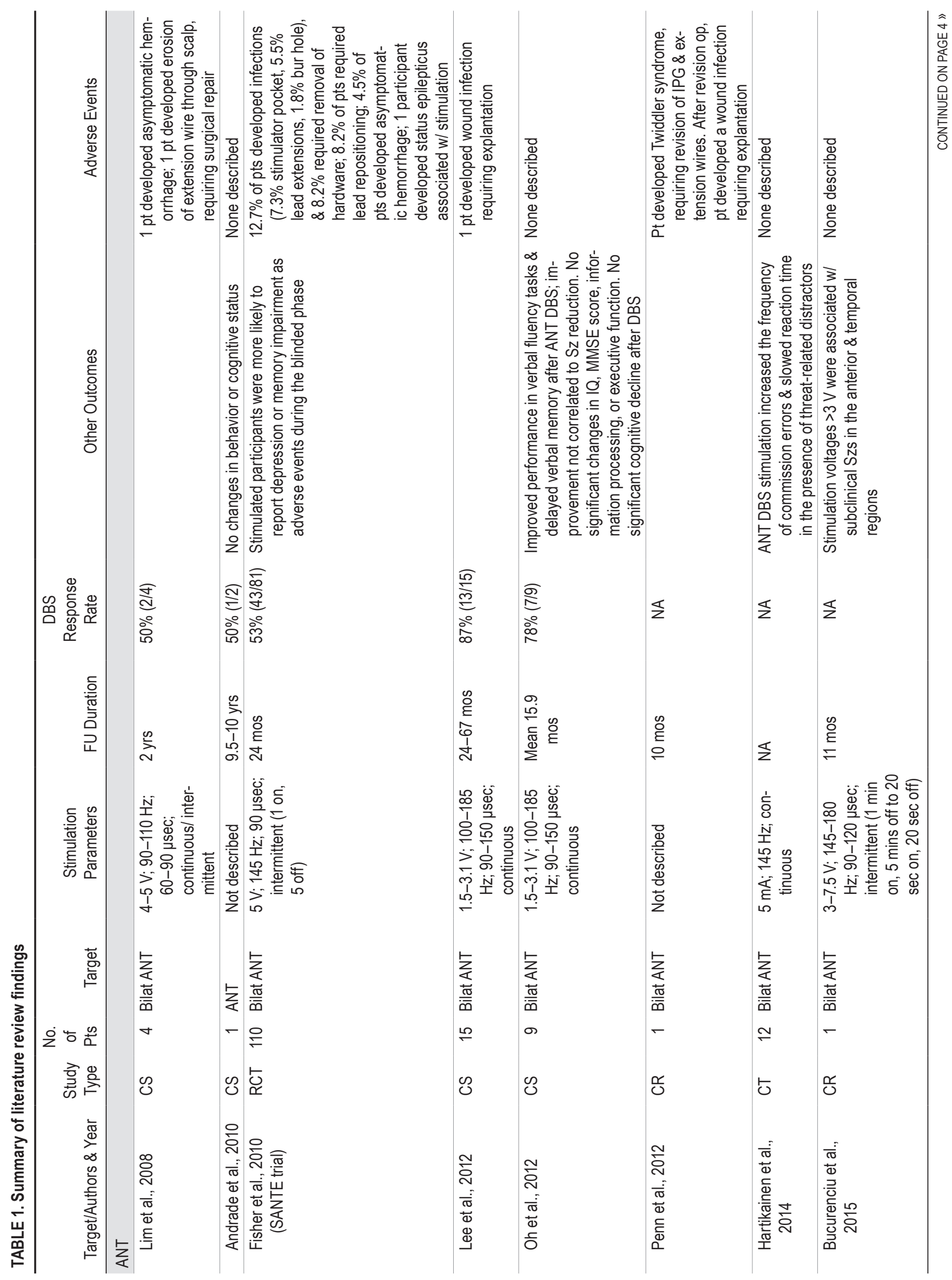




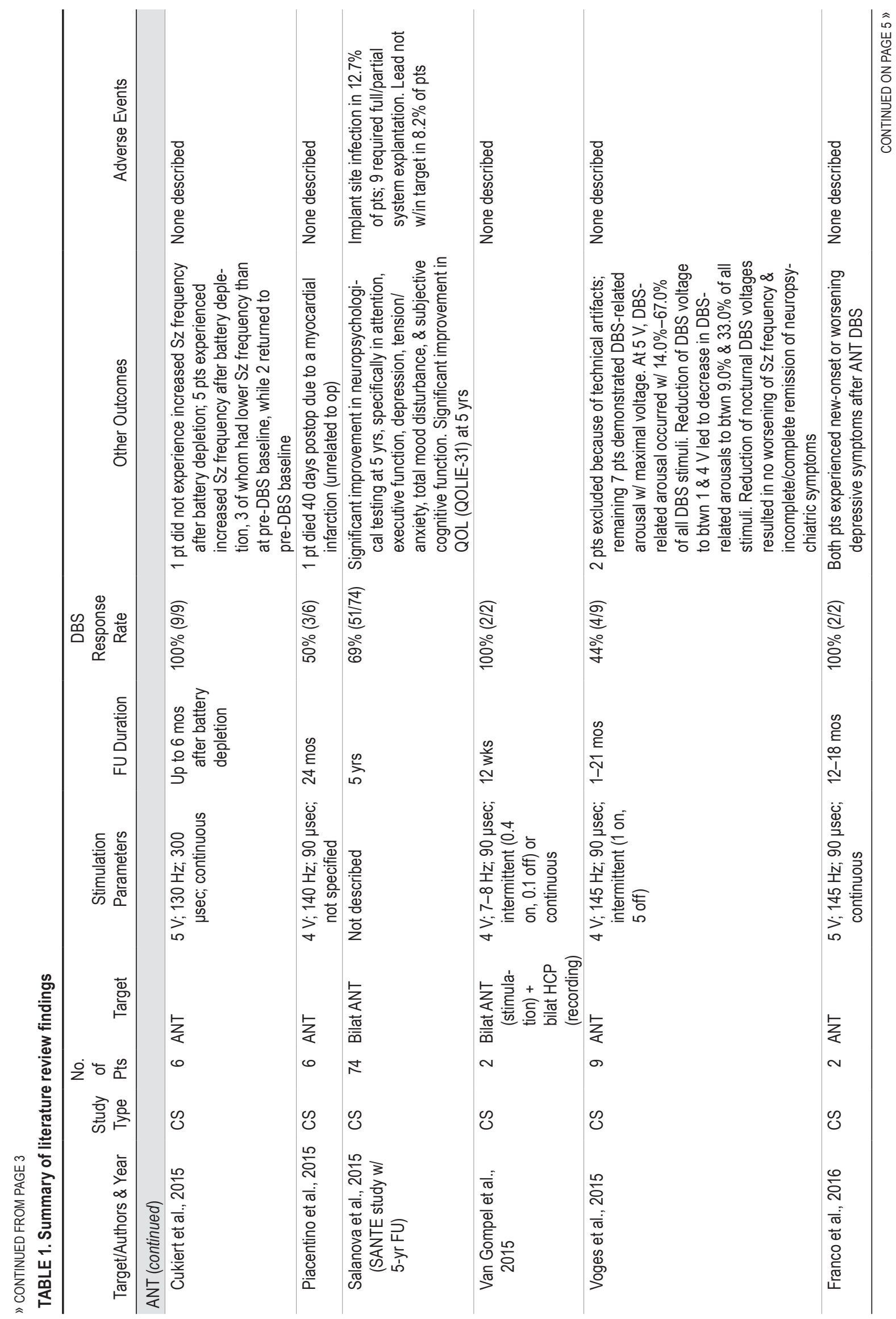




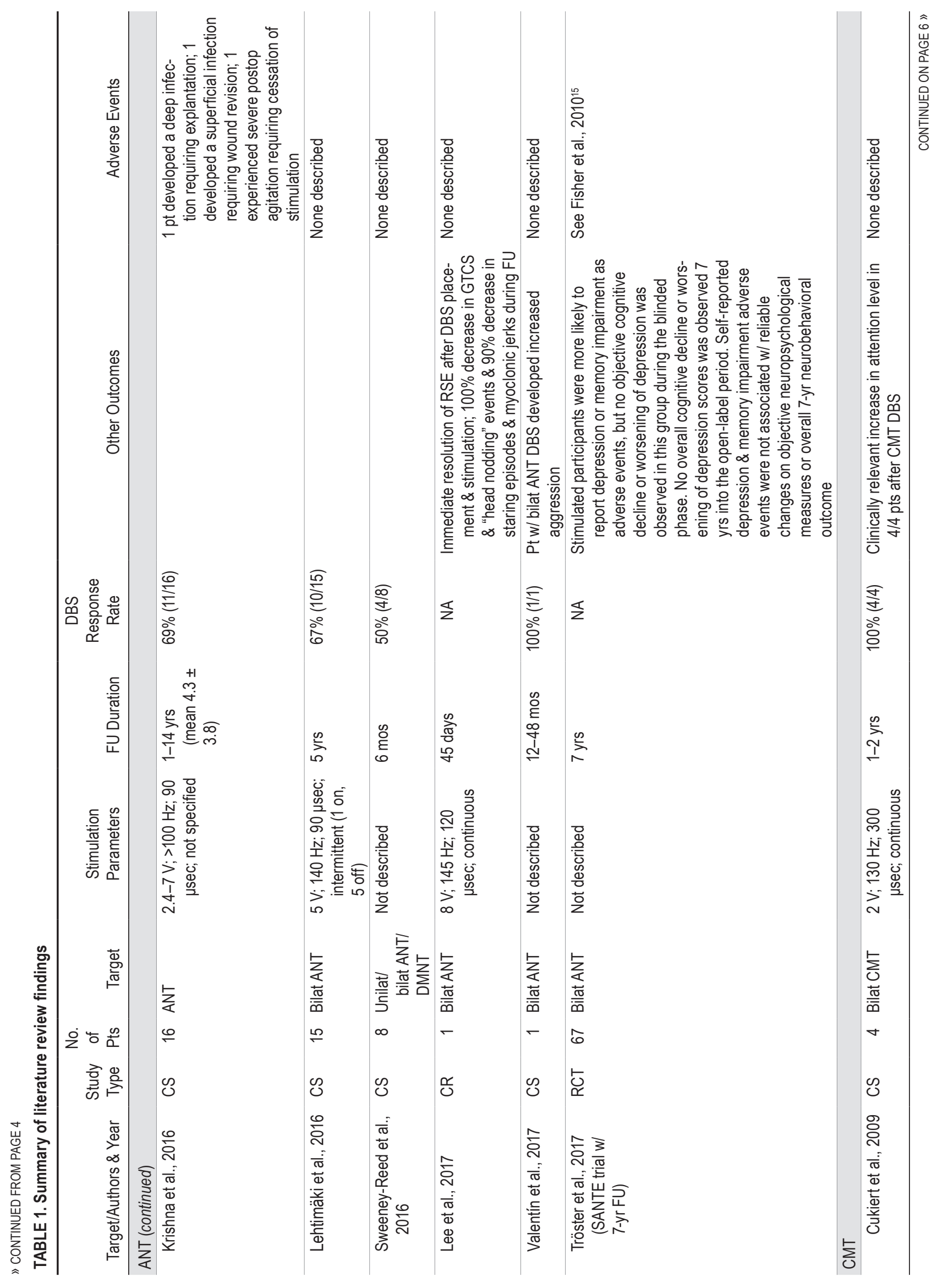

Neurosurg Focus Volume 45 • August 2018 


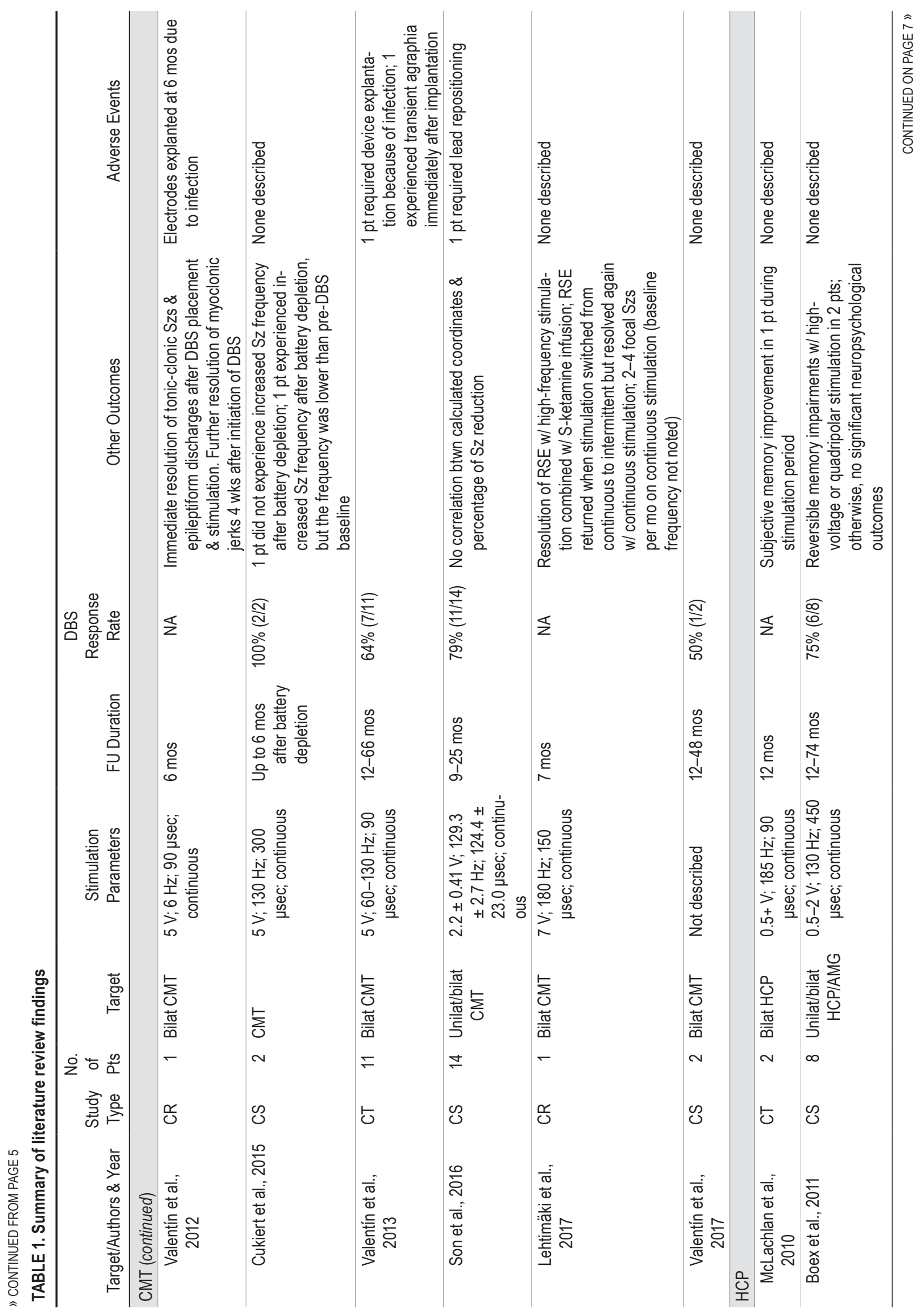


Zhou et al.

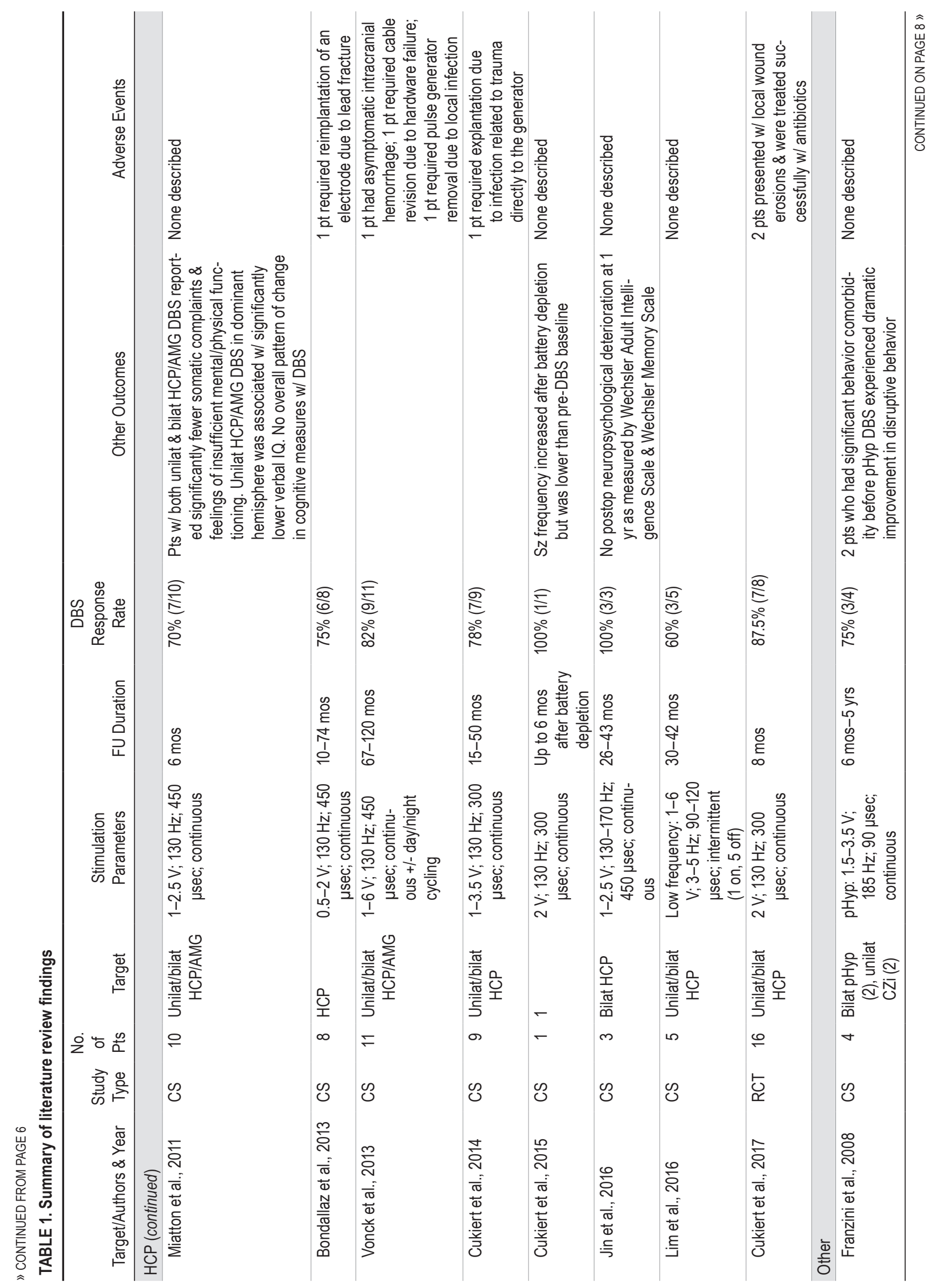




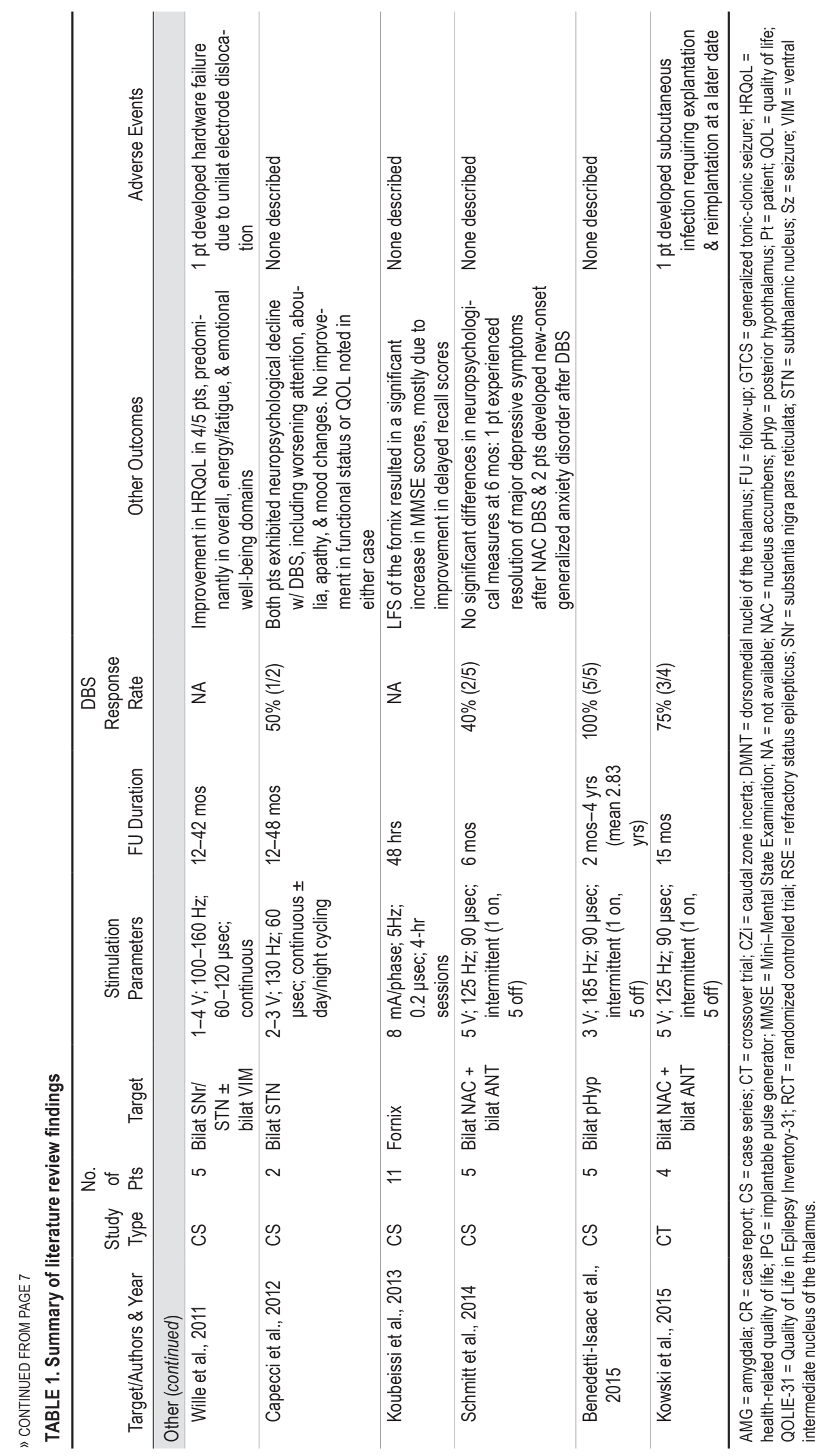


tient who experienced at least a $50 \%$ reduction in seizure frequency with DBS.

\section{Anterior Nucleus of the Thalamus \\ Seizure Outcomes}

Interest in ANT DBS for epilepsy has increased substantially since Kerrigan et al. ${ }^{21}$ published an open-label trial demonstrating its efficacy in 2004. Over the past decade, 20 studies have been published, reporting on 220 patients who underwent ANT DBS for the treatment of epilepsy. 1,5,11,15,16,19,25,29,30,32,34,37-40,48,49,53-55 These studies have reported responder rates ranging from $44 \%$ to $100 \%$. Patients from the largest series $(n=110)$ were reported to have median seizure reduction rates of $56 \%$ at 2 years $^{15}$ and $69 \%$ at 5 years. ${ }^{40}$ The Stimulation of the Anterior $\mathrm{Nu}-$ cleus of the Thalamus for Epilepsy (SANTE) trial was the first randomized controlled trial investigating ANT DBS for the treatment of intractable epilepsy. As described by Fisher et al. ${ }^{15}$ in 2010, a total of 110 patients underwent bilateral ANT DBS, of whom 109 were then randomized to receive stimulation or no stimulation during a 3-month blinded phase. Patients randomized to stimulation experienced a $29 \%$ greater reduction in seizures compared to the control group $(\mathrm{p}=0.002)$; median declines at the end of the blinded phase were $40.4 \%$ and $14.5 \%$, respectively. After the 3-month blinded phase, all patients received stimulation as part of an open-ended unblinded phase. At 2-year follow-up, the authors reported a median 56\% reduction in seizure frequency, with a $54 \%$ response rate. Overall, 808 adverse events were documented during the first 13 months of follow-up, 55 of which were considered serious. Implant site infection developed in 14 participants (12.7\%), 9 of whom required full or partial removal of the hardware. Five hemorrhages (4.5\%) were detected incidentally on imaging, none of which were symptomatic. In 2015 , Salanova et al. ${ }^{40}$ published 5 -year outcomes of the SANTE trial patients $(\mathrm{n}=74)$ and found a median $69 \%$ reduction in seizure frequency, with $68 \%$ of patients classified as responders.

While the SANTE trial is the only randomized controlled trial on ANT DBS for epilepsy, several other studies have evaluated the safety and efficacy of this therapy. In 2008, Lim et al. ${ }^{34}$ reported a mean $49.6 \%$ reduction in seizures with stimulation (range 35\%-61\%) at the 2-year follow-up for 4 patients. One patient achieved complete remission, and 2 other patients were responders, defined as a seizure reduction rate $\geq 60 \%$. However, the complication rate was $50 \%$. One patient developed a frontal hematoma and transient left-sided weakness, and a second patient developed extension lead erosion through the scalp that ultimately required removal of the entire DBS system. In 2012 , Lee et al. ${ }^{30}$ monitored 15 patients for an average of 27 months and noted a mean $70.4 \%$ reduction in seizure frequency; 13 of the 15 patients (87\%) experienced $>50 \%$ reduction in seizures. The authors reported that only one major adverse event occurred in a patient who developed a wound infection requiring system explantation.

In 2015, Cukiert et al. ${ }^{11}$ studied the effects of battery depletion on seizure frequency in 6 patients treated with ANT DBS for intractable epilepsy. They demonstrated that when battery depletion occurred after 3 years of continu- ous ANT DBS, 5 patients experienced increased seizure frequency and 1 did not. Of the 5 patients who experienced an increase in seizure frequency after battery depletion, 3 patients still had a lower seizure rate than they did at their pre-DBS baseline, whereas 2 patients returned to their pre-DBS baseline seizure rate. The authors posited that, in those patients who did not experience a full return to pre-DBS baseline seizure frequency, some degree of network neuromodulation may have occurred with prolonged continuous DBS.

\section{Neuropsychological and Cognitive Outcomes}

Although patients enrolled in the SANTE trial exhibited no objective differences in neuropsychological testing for cognition or mood during the blinded phase of the study, significantly more patients reported subjective adverse events related to depression $(\mathrm{p}=0.02)$ and memory impairment $(\mathrm{p}=0.03) .{ }^{15}$ Gradual improvement in neuropsychological outcomes was noted at the 5-year follow-up, specifically in attention, executive function, depression, tension/anxiety, total mood disturbance, and subjective cognitive function. ${ }^{40}$ At 7-year follow-up, Tröster et al. ${ }^{49}$ noted that no patients $(n=67)$ experienced significant worsening of cognition or depression on objective testing. Patients achieved significantly higher scores on objective measures of executive function and attention. They also noted that self-reported depression and memory-related adverse events were not associated with declines in neuropsychiatric testing.

In 2012, Oh et al. ${ }^{37}$ reported 12-month cognitive and behavioral outcomes after bilateral ANT DBS for epilepsy in 9 patients. They found significant improvements in verbal fluency tasks $(\mathrm{p}<0.05)$ and delayed verbal memory ( $\mathrm{p}$ $=0.017$ ) but saw no overall change in general measures of intelligence, information processing, and executive functioning. There was also no evidence of significant cognitive decline 1 year after ANT DBS. They postulated that cognition may have improved directly through stimulation of the medial temporal structures via the Papez circuit and indirectly through reductions in seizure frequency and antiepileptic drug use.

In 2014, Hartikainen et al. ${ }^{19}$ studied the effects of threatrelated emotional distractors and rule switching in 12 patients using a computer-based executive function test and found that stimulation increased commission error frequency and slowed participant reaction times in the presence of threat- or emotion-related distractors. They speculated that ANT DBS affected response inhibition and emotional reactivity modulation through indirect stimulation of limbic structures such as the nucleus accumbens and orbitomedial prefrontal cortex via the Papez circuit.

\section{Surgical Considerations}

As ANT DBS has become an increasingly accepted therapy for epilepsy, interest has focused on refining targeting and patient selection. Van Gompel et al. ${ }^{54}$ recently reported on 2 such novel targeting techniques. First, electrodes were placed along a posterior inferior parietal route, to avoid intraventricular hemorrhage and lead misplacement associated with transventricular and lateral transcortical approaches. Second, they used recordings from 
concomitantly placed hippocampal electrodes to verify accurate lead placement. Both patients in this series experienced $>50 \%$ reduction in seizures over a 3-month follow-up period, and no adverse events, including lead misplacement, were reported.

Piacentino et al. ${ }^{39}$ classified 6 patients based on preoperative imaging and continuous video electroencephalography, and they demonstrated that ANT DBS was most effective in patients with epileptic origins strictly in the limbic system who had no discrete anatomical lesions. Lehtimäki et al. ${ }^{32}$ analyzed the placement of 62 contacts in 15 patients, 10 of whom were responders. Using an ANTnormalized coordinate system, they found that contacts in responders were placed significantly more anteriorly and superiorly than they were in nonresponders. They hypothesized that the white matter structures at the inferior and posterior aspects of the ANT prevented the spread of stimulation current into the ANT, which limited the utility of electrodes placed in that region. Krishna et al. ${ }^{25}$ found similar results, noting that patients with the most long-term stimulation benefit had electrodes placed in the anteroventral ANT in close proximity to the mammillothalamic tract.

\section{Centromedian Nucleus of the Thalamus}

Seven studies over the past decade have investigated the use of CMT DBS for the treatment of epilepsy in 35 patients. ${ }^{10,11,31,44,51-53}$ Treatment response rates range from $50 \%$ to $100 \%$, depending on the study and the type of epilepsy being treated. The largest series, published by Son et al. in 2016, ${ }^{44}$ reported a $79 \%$ response rate (11 of 14 patients), with a mean seizure frequency reduction of $68 \%$. They did not find any correlation between lead positioning and the magnitude of seizure reduction on regression analysis.

In 2009, Cukiert et al. ${ }^{10}$ evaluated the use of CMT DBS for refractory generalized epilepsy in 4 patients who previously underwent corpus callosotomies. At 1 to 2 years of follow-up, seizure reduction rates ranged from $65 \%$ to $95 \%$. No significant morbidity or mortality was reported other than transient contralateral paresthesia. Valentín and colleagues ${ }^{51}$ published results from a single-blinded, nonrandomized, crossover trial of 11 patients with generalized or frontal epilepsy who were treated with bilateral CMT DBS in which they found that CMT DBS was more effective in treating generalized epilepsy than frontal lobe epilepsy. In this study, $40 \%(2 / 5)$ of patients with frontal lobe epilepsy and 83\% (5/6) of patients with generalized epilepsy responded to DBS therapy, for a total overall response rate of $64 \%(7 / 11)$.

Two case reports of CMT DBS used in patients with status epilepticus have been published. In 2012, Valentín et al. ${ }^{52}$ described the successful use of CMT DBS to treat a 27-year-old patient with 5-week history of refractory status epilepticus. Electroencephalography findings were consistent with resolution of status epilepticus on initiation of low-frequency 6-Hz CMT DBS. The patient developed a wound infection 6 months postprocedure and required device explantation; however, symptoms reportedly did not recur. In 2017, Lehtimäki et al. ${ }^{31}$ reported the case of a 17-year-old male patient with refractory status epilepticus thought to be secondary to common variable immunodeficiency-associated encephalomyelitis. Bilateral high-frequency $180-\mathrm{Hz}$ DBS, coupled with a continuous S-ketamine infusion, proved effective in terminating the status epilepticus. However, all attempts to wean the patient from continuous to intermittent stimulation resulted in relapse.

\section{Hippocampus}

Over the past decade, 10 studies have been published on the use of HCP DBS for the treatment of epilepsy in 73 patients. ${ }^{3,4,11,12,13,20,33,35,36,56}$ The reported response rates have ranged from $60 \%$ to $100 \%$.

In 2014, Cukiert and colleagues ${ }^{12}$ published the results of a prospective study investigating the effects of unilateral or bilateral HCP DBS in 9 consecutive patients with refractory temporal lobe epilepsy (TLE). At a mean follow-up of 30.1 months, 2 of 2 patients with unilateral temporal lobe sclerosis responded to unilateral DBS therapy, with a mean reduction in seizure frequency of $78 \%$. Three of 4 patients with bilateral mesial temporal lobe sclerosis responded to unilateral stimulation, whereas 1 patient did not respond even when advanced to bilateral stimulation; these patients experienced a mean reduction in seizure frequency of $63 \%$. Similarly, 2 of 3 patients with normal MRI results responded to unilateral DBS therapy, whereas 1 did not respond to both unilateral and bilateral stimulation; these patients experienced a mean reduction in seizure frequency of 58\%. One patient in the study developed a wound infection related to a direct impact to the stimulator and subsequently required device explantation; otherwise, no adverse events were reported. Cukiert and colleagues ${ }^{13}$ then reported in 2017 on the results of a prospective, double-blind, randomized controlled trial evaluating the efficacy of unilateral and bilateral HCP DBS in 16 patients with refractory TLE. Two months after surgery, all patients were randomized to stimulation on or off for a 6-month blinded period. The authors reported that, of the 8 patients randomized to the on-stimulation group, 4 became seizure free and 7 were defined as responders, whereas 1 patient did not respond to DBS therapy. The investigators further noted that the experimental group experienced significantly fewer simple partial and complex partial seizures than the control group throughout the blinded period. Two patients with superficial wound infections were successfully treated with antibiotics, and no other complications were noted.

Vonck et al. ${ }^{56}$ reported on 11 patients who underwent bilateral HCP DBS electrode implantation, with stimulation laterality applied based on seizure localization. After 2.5-3 years of follow-up, patients who were initially started on unilateral stimulation were converted to bilateral stimulation if seizure reduction of $>90 \%$ had not been achieved. At final follow-up, and after switching to bilateral stimulation as necessary, 6 patients achieved $\geq 90 \%$ seizure reduction, 3 patients achieved seizure reduction rates ranging from $40 \%$ to $70 \%$, and 2 patients achieved $<30 \%$ seizure reduction. Importantly, the authors found that switching from unilateral to bilateral stimulation further improved seizure outcomes in 3 of 5 patients with unilateral ictal onset. Implementing day-night cycling after attaining treat- 
ment stability did not affect seizure control, and no changes in neuropsychological testing were noted after DBS therapy. One patient developed an asymptomatic intracranial hemorrhage diagnosed incidentally on imaging, 1 patient required cable revision because of hardware failure, and 1 patient developed a wound infection requiring system explantation; otherwise, no serious adverse events were reported.

\section{Discussion}

The role of neuromodulation for the treatment of epilepsy has expanded over the past decade. Vagal nerve stimulation and responsive neurostimulation are approved therapies for epilepsy in the US, and DBS is available in Canada and Europe. To date, the most thoroughly investigated targets in DBS for epilepsy are the ANT, CMT, and $\mathrm{HCP}$, with responder rates of 44\%-100\%, 50\%-100\%, and 60\%-100\%, respectively. Other alternative targets have demonstrated varying degrees of success. Level I evidence from the SANTE trial demonstrates that response rates and seizure-freedom rates of $53 \%$ and $13 \%$, respectively, can be achieved with ANT stimulation (2 years after stimulation), ${ }^{15}$ with complication rates comparable to those of other large DBS series for movement disorders. ${ }^{7,18,43}$ In contrast to many neuromodulation studies for other nonmovement disorder indications, long-term follow-up data are available for this cohort of patients, which supports the safety durability of the therapy over time. Five years after stimulation initiation, a $69 \%$ DBS response rate was achieved $^{40,49}$ and no deleterious effects on neuropsychological outcomes were documented. ${ }^{40,49}$ It must be noted, however, that long-term follow-up was obtained only for 74 of the original 110 patients. Additional nonrandomized studies have demonstrated a $44 \%$ to $100 \%$ seizure response rate. ${ }^{1,5,11,16,19,25,29,30,32,34,37-39,48,54,55}$ Recent innovations in implantation technique, such as the posterior inferior parietal electrode trajectory and concomitant hippocampal electrode placement, warrant additional research and consideration for widespread implementation. ${ }^{25,32,54}$

CMT DBS has also demonstrated promising results in the treatment of patients with generalized epilepsy. ${ }^{10,51}$ Some studies suggest that patients with generalized epilepsy respond better to CMT DBS than patients with frontal lobe epilepsy; however, the data are still conflicting, and more research is needed to determine whether this is true..$^{0,51,53}$ Overall, most evidence for CMT DBS has come from Level III and IV studies. ${ }^{10,51}$ Studies with higher levels of evidence and with larger patient cohorts and longer follow-up are necessary to determine whether CMT is truly effective in treating the target indications.

Evidence is conflicting in reports on a small number of patients regarding the efficacy of HCP DBS for epilepsy. Response rates range from $78 \%$ to $100 \%$, depending on epilepsy etiology, ${ }^{12,13,20}$ and a recently published randomized controlled trial of 16 patients demonstrated that patients who received HCP DBS experienced significantly fewer seizures than controls. ${ }^{13}$ However, data from a crossover study published by McLachlan et al. ${ }^{35}$ suggested that HCP DBS was clinically ineffective at treating seizures in 2 patients who had refractory TLE. Recent advances in surgical approaches, including the use of bilateral stimulation in patients with unilateral TLE and the optimization of targeting parameters, ${ }^{4,56}$ warrant further clinical study to determine whether outcomes are improved with these modifications. Investigation of other targets, such as the posterior hypothalamus, nucleus accumbens, and subthalamic nucleus or substantia nigra pars reticulata, is based on small case series and reports and is still in very early and preliminary phases. No definitive conclusions can currently be drawn regarding the efficacy of these targets in treating epilepsy.

Despite the enormous amount of research that has been conducted on DBS for the treatment of epilepsy, countless avenues of inquiry remain open for further investigation. For example, a great deal of uncertainty remains about the efficacy of many of the obscure targets, such as the subthalamic nucleus, nucleus accumbens, and posterior hypothalamus. More data on patient outcomes after DBS of these targets, even in the form of nonrandomized, retrospective case series, may provide more information about whether continued pursuit of these targets is worthwhile. For targets for which outcomes are well defined, such as the ANT, future research may focus on further refining patient selection, targeting, and stimulation parameters, in a manner similar to that in studies conducted by Voges et al., ${ }^{55}$ Van Gompel et al., ${ }^{54}$ and Lehtimäki et al. ${ }^{32}$ Finally, although high-quality, nonrandomized, prospective evidence exists to support the efficacy of CMT DBS for epilepsy, a well-powered, randomized controlled trial is still necessary to establish the clinical efficacy of this target.

\section{Conclusions}

The investigation of DBS for the treatment of refractory epilepsy has seen substantial growth and progress in the past decade. Level I evidence supports the efficacy of ANT DBS to treat patients with refractory epilepsy, which has demonstrated continued therapeutic success up to 7 years after surgery. Investigators continue to gather supporting evidence for the safety and efficacy over time of CMT and HCP as stimulation targets, and additional evidence and further study are required for newer targets, such as the nucleus accumbens and posterior hypothalamus, before they are considered for broader therapeutic use.

\section{Acknowledgments}

We thank the staff of Neuroscience Publications at Barrow Neurological Institute for assistance with manuscript preparation.

\section{References}

1. Andrade DM, Hamani C, Lozano AM, Wennberg RA: Dravet syndrome and deep brain stimulation: seizure control after 10 years of treatment. Epilepsia 51:1314-1316, 2010

2. Benedetti-Isaac JC, Torres-Zambrano M, Vargas-Toscano A, Perea-Castro E, Alcalá-Cerra G, Furlanetti LL, et al: Seizure frequency reduction after posteromedial hypothalamus deep brain stimulation in drug-resistant epilepsy associated with intractable aggressive behavior. Epilepsia 56:1152-1161, 2015

3. Boëx C, Seeck M, Vulliémoz S, Rossetti AO, Staedler C, Spinelli L, et al: Chronic deep brain stimulation in mesial temporal lobe epilepsy. Seizure 20:485-490, 2011 
4. Bondallaz P, Boëx C, Rossetti AO, Foletti G, Spinelli L, Vulliemoz $\mathrm{S}$, et al: Electrode location and clinical outcome in hippocampal electrical stimulation for mesial temporal lobe epilepsy. Seizure 22:390-395, 2013

5. Bucurenciu I, Staack AM, Hubbard I, Rona S, Gharabaghi A, Steinhoff BJ: Coincidence of non-convulsive epileptic seizures and electrical stimulation of thalamic anterior nuclei in an epileptic patient. Clin Neurophysiol 126:1054-1056, 2015

6. Capecci M, Ricciuti RA, Ortenzi A, Paggi A, Durazzi V, Rychlicki F, et al: Chronic bilateral subthalamic stimulation after anterior callosotomy in drug-resistant epilepsy: longterm clinical and functional outcome of two cases. Epilepsy Res 98:135-139, 2012

7. Chen T, Mirzadeh Z, Chapple K, Lambert M, Ponce FA: Complication rates, lengths of stay, and readmission rates in "awake" and "asleep" deep brain simulation. J Neurosurg 127:360-369, 2017

8. Cooper IS, Amin I, Gilman S: The effect of chronic cerebellar stimulation upon epilepsy in man. Trans Am Neurol Assoc 98:192-196, 1973

9. Cooper IS, Amin I, Riklan M, Waltz JM, Poon TP: Chronic cerebellar stimulation in epilepsy: clinical and anatomical studies. Arch Neurol 33:559-570, 1976

10. Cukiert A, Burattini JA, Cukiert CM, Argentoni-Baldochi M, Baise-Zung C, Forster CR, et al: Centro-median stimulation yields additional seizure frequency and attention improvement in patients previously submitted to callosotomy. Seizure 18:588-592, 2009

11. Cukiert A, Cukiert CM, Burattini JA, Lima Ade M: Seizure outcome after battery depletion in epileptic patients submitted to deep brain stimulation. Neuromodulation 18:439441,2015

12. Cukiert A, Cukiert CM, Burattini JA, Lima AM: Seizure outcome after hippocampal deep brain stimulation in a prospective cohort of patients with refractory temporal lobe epilepsy. Seizure 23:6-9, 2014

13. Cukiert A, Cukiert CM, Burattini JA, Mariani PP, Bezerra DF: Seizure outcome after hippocampal deep brain stimulation in patients with refractory temporal lobe epilepsy: a prospective, controlled, randomized, double-blind study. Epilepsia 58:1728-1733, 2017

14. Englot DJ, Birk H, Chang EF: Seizure outcomes in nonresective epilepsy surgery: an update. Neurosurg Rev 40:181-194, 2017

15. Fisher R, Salanova V, Witt T, Worth R, Henry T, Gross R, et al: Electrical stimulation of the anterior nucleus of thalamus for treatment of refractory epilepsy. Epilepsia 51:899-908, 2010

16. Franco A, Pimentel J, Campos AR, Morgado C, Pinelo S, Ferreira AG, et al: Stimulation of the bilateral anterior nuclei of the thalamus in the treatment of refractory epilepsy: two cases of subcortical band heterotopia. Epileptic Disord 18:426-430, 2016

17. Franzini A, Messina G, Marras C, Villani F, Cordella R, Broggi G: Deep brain stimulation of two unconventional targets in refractory non-resectable epilepsy. Stereotact Funct Neurosurg 86:373-381, 2008

18. Hamani C, Lozano AM: Hardware-related complications of deep brain stimulation: a review of the published literature. Stereotact Funct Neurosurg 84:248-251, 2006

19. Hartikainen KM, Sun L, Polvivaara M, Brause M, Lehtimäki $\mathrm{K}$, Haapasalo J, et al: Immediate effects of deep brain stimulation of anterior thalamic nuclei on executive functions and emotion-attention interaction in humans. J Clin Exp Neuropsychol 36:540-550, 2014

20. Jin H, Li W, Dong C, Wu J, Zhao W, Zhao Z, et al: Hippocampal deep brain stimulation in nonlesional refractory mesial temporal lobe epilepsy. Seizure 37:1-7, 2016

21. Kerrigan JF, Litt B, Fisher RS, Cranstoun S, French JA, Blum
DE, et al: Electrical stimulation of the anterior nucleus of the thalamus for the treatment of intractable epilepsy. Epilepsia 45:346-354, 2004

22. Klinger NV, Mittal S: Clinical efficacy of deep brain stimulation for the treatment of medically refractory epilepsy. Clin Neurol Neurosurg 140:11-25, 2016

23. Koubeissi MZ, Kahriman E, Syed TU, Miller J, Durand DM: Low-frequency electrical stimulation of a fiber tract in temporal lobe epilepsy. Ann Neurol 74:223-231, 2013

24. Kowski AB, Voges J, Heinze HJ, Oltmanns F, Holtkamp M, Schmitt FC: Nucleus accumbens stimulation in partial epilepsy - a randomized controlled case series. Epilepsia 56:e78-e82, 2015

25. Krishna V, King NK, Sammartino F, Strauss I, Andrade DM, Wennberg RA, et al: Anterior nucleus deep brain stimulation for refractory epilepsy: insights into patterns of seizure control and efficacious target. Neurosurgery 78:802-811, 2016

26. Kwan P, Brodie MJ: Early identification of refractory epilepsy. N Engl J Med 342:314-319, 2000

27. Kwan P, Sperling MR: Refractory seizures: try additional antiepileptic drugs (after two have failed) or go directly to early surgery evaluation? Epilepsia 50 (Suppl 8):57-62, 2009

28. Laxpati NG, Kasoff WS, Gross RE: Deep brain stimulation for the treatment of epilepsy: circuits, targets, and trials. Neurotherapeutics 11:508-526, 2014

29. Lee CY, Lim SN, Wu T, Lee ST: Successful treatment of refractory status epilepticus using anterior thalamic nuclei deep brain stimulation. World Neurosurg 99:14-18, 2017

30. Lee KJ, Shon YM, Cho CB: Long-term outcome of anterior thalamic nucleus stimulation for intractable epilepsy. Stereotact Funct Neurosurg 90:379-385, 2012

31. Lehtimäki K, Långsjö JW, Ollikainen J, Heinonen H, Möttönen T, Tähtinen T, et al: Successful management of superrefractory status epilepticus with thalamic deep brain stimulation. Ann Neurol 81:142-146, 2017

32. Lehtimäki K, Möttönen T, Järventausta K, Katisko J, Tähtinen T, Haapasalo J, et al: Outcome based definition of the anterior thalamic deep brain stimulation target in refractory epilepsy. Brain Stimul 9:268-275, 2016

33. Lim SN, Lee CY, Lee ST, Tu PH, Chang BL, Lee CH, et al: Low and high frequency hippocampal stimulation for drugresistant mesial temporal lobe epilepsy. Neuromodulation 19:365-372, 2016

34. Lim SN, Lee ST, Tsai YT, Chen IA, Tu PH, Chen JL, et al: Long-term anterior thalamus stimulation for intractable epilepsy. Chang Gung Med J 31:287-296, 2008

35. McLachlan RS, Pigott S, Tellez-Zenteno JF, Wiebe S, Parrent A: Bilateral hippocampal stimulation for intractable temporal lobe epilepsy: impact on seizures and memory. Epilepsia 51:304-307, 2010

36. Miatton M, Van Roost D, Thiery E, Carrette E, Van Dycke A, Vonck K, et al: The cognitive effects of amygdalohippocampal deep brain stimulation in patients with temporal lobe epilepsy. Epilepsy Behav 22:759-764, 2011

37. Oh YS, Kim HJ, Lee KJ, Kim YI, Lim SC, Shon YM: Cognitive improvement after long-term electrical stimulation of bilateral anterior thalamic nucleus in refractory epilepsy patients. Seizure 21:183-187, 2012

38. Penn DL, Wu C, Skidmore C, Sperling MR, Sharan AD: Twiddler's syndrome in a patient with epilepsy treated with deep brain stimulation. Epilepsia 53:e119-e121, 2012

39. Piacentino M, Durisotti C, Garofalo PG, Bonanni P, Volzone A, Ranzato F, et al: Anterior thalamic nucleus deep brain Stimulation (DBS) for drug-resistant complex partial seizures (CPS) with or without generalization: long-term evaluation and predictive outcome. Acta Neurochir (Wien) 157:15251532,2015

40. Salanova V, Witt T, Worth R, Henry TR, Gross RE, Nazzaro $\mathrm{JM}$, et al: Long-term efficacy and safety of thalamic stimula- 
tion for drug-resistant partial epilepsy. Neurology 84:10171025,2015

41. Sander JW: The epidemiology of epilepsy revisited. Curr Opin Neurol 16:165-170, 2003

42. Schmitt FC, Voges J, Heinze HJ, Zaehle T, Holtkamp M, Kowski AB: Safety and feasibility of nucleus accumbens stimulation in five patients with epilepsy. J Neurol 261:14771484, 2014

43. Sillay KA, Larson PS, Starr PA: Deep brain stimulator hardware-related infections: incidence and management in a large series. Neurosurgery 62:360-367, 2008

44. Son BC, Shon YM, Choi JG, Kim J, Ha SW, Kim SH, et al: Clinical outcome of patients with deep brain stimulation of the centromedian thalamic nucleus for refractory epilepsy and location of the active contacts. Stereotact Funct Neurosurg 94:187-197, 2016

45. Sperling MR: The consequences of uncontrolled epilepsy. CNS Spectr 9:98-101, 106-109, 2004

46. Sprengers M, Vonck K, Carrette E, Marson AG, Boon P: Deep brain and cortical stimulation for epilepsy. Cochrane Database Syst Rev (6):CD008497, 2014

47. Sprengers M, Vonck K, Carrette E, Marson AG, Boon P: Deep brain and cortical stimulation for epilepsy. Cochrane Database Syst Rev 7:CD008497, 2017

48. Sweeney-Reed CM, Lee H, Rampp S, Zaehle T, Buentjen L, Voges J, et al: Thalamic interictal epileptiform discharges in deep brain stimulated epilepsy patients. J Neurol 263:21202126, 2016

49. Tröster AI, Meador KJ, Irwin CP, Fisher RS: Memory and mood outcomes after anterior thalamic stimulation for refractory partial epilepsy. Seizure 45:133-141, 2017

50. Upton AR, Cooper IS, Springman M, Amin I: Suppression of seizures and psychosis of limbic system origin by chronic stimulation of anterior nucleus of the thalamus. Int $\mathbf{J}$ Neurol 19-20:223-230, 1985-1986

51. Valentín A, García Navarrete E, Chelvarajah R, Torres C, Navas M, Vico L, et al: Deep brain stimulation of the centromedian thalamic nucleus for the treatment of generalized and frontal epilepsies. Epilepsia 54:1823-1833, 2013

52. Valentín A, Nguyen HQ, Skupenova AM, Agirre-Arrizubieta Z, Jewell S, Mullatti N, et al: Centromedian thalamic nuclei deep brain stimulation in refractory status epilepticus. Brain Stimul 5:594-598, 2012

53. Valentín A, Selway RP, Amarouche M, Mundil N, Ughratdar
I, Ayoubian L, et al: Intracranial stimulation for children with epilepsy. Eur J Paediatr Neurol 21:223-231, 2017

54. Van Gompel JJ, Klassen BT, Worrell GA, Lee KH, Shin C, Zhao CZ, et al: Anterior nuclear deep brain stimulation guided by concordant hippocampal recording. Neurosurg Focus 38(6):E9, 2015

55. Voges BR, Schmitt FC, Hamel W, House PM, Kluge C, Moll CK, et al: Deep brain stimulation of anterior nucleus thalami disrupts sleep in epilepsy patients. Epilepsia 56:e99-e103, 2015

56. Vonck K, Sprengers M, Carrette E, Dauwe I, Miatton M, Meurs A, et al: A decade of experience with deep brain stimulation for patients with refractory medial temporal lobe epilepsy. Int J Neural Syst 23:1250034, 2013

57. Wille C, Steinhoff BJ, Altenmüller DM, Staack AM, Bilic S, Nikkhah G, et al: Chronic high-frequency deep-brain stimulation in progressive myoclonic epilepsy in adulthood-report of five cases. Epilepsia 52:489-496, 2011

58. World Health Organization: Epilepsy. WHO.int. February 8, 2018 (http://www.who.int/en/news-room/fact-sheets/detail/ epilepsy) [Accessed May 30, 2018]

\section{Disclosures}

The authors report no conflict of interest concerning the materials or methods used in this study or the findings specified in this paper.

\section{Author Contributions}

Conception and design: Ponce, Zhou, Chen, Shetter. Acquisition of data: Zhou, Farber. Analysis and interpretation of data: Zhou, Farber. Drafting the article: Zhou, Farber. Critically revising the article: Ponce, Zhou, Chen, Shetter. Reviewed submitted version of manuscript: Ponce, Zhou, Shetter. Statistical analysis: Zhou. Administrative/technical/material support: Zhou. Study supervision: Ponce, Shetter.

\section{Correspondence}

Francisco A. Ponce: Barrow Neurological Institute, St. Joseph's Hospital and Medical Center, Phoenix, AZ.neuropub@ barrowneuro.org. 